Document downloaded from:

http://hdl.handle.net/10251/49177

This paper must be cited as:

Capilla Roma, R.; Martín Furones, AE.; Anquela Julián, AB.; Berné Valero, JL. (2012). Frame transformation and geoid undulation transfer to GNSS real time positions through the new RTCM 3.1 transformation messages. Survey Review. 44(324):30-36. doi:10.1179/1752270611Y.0000000010.

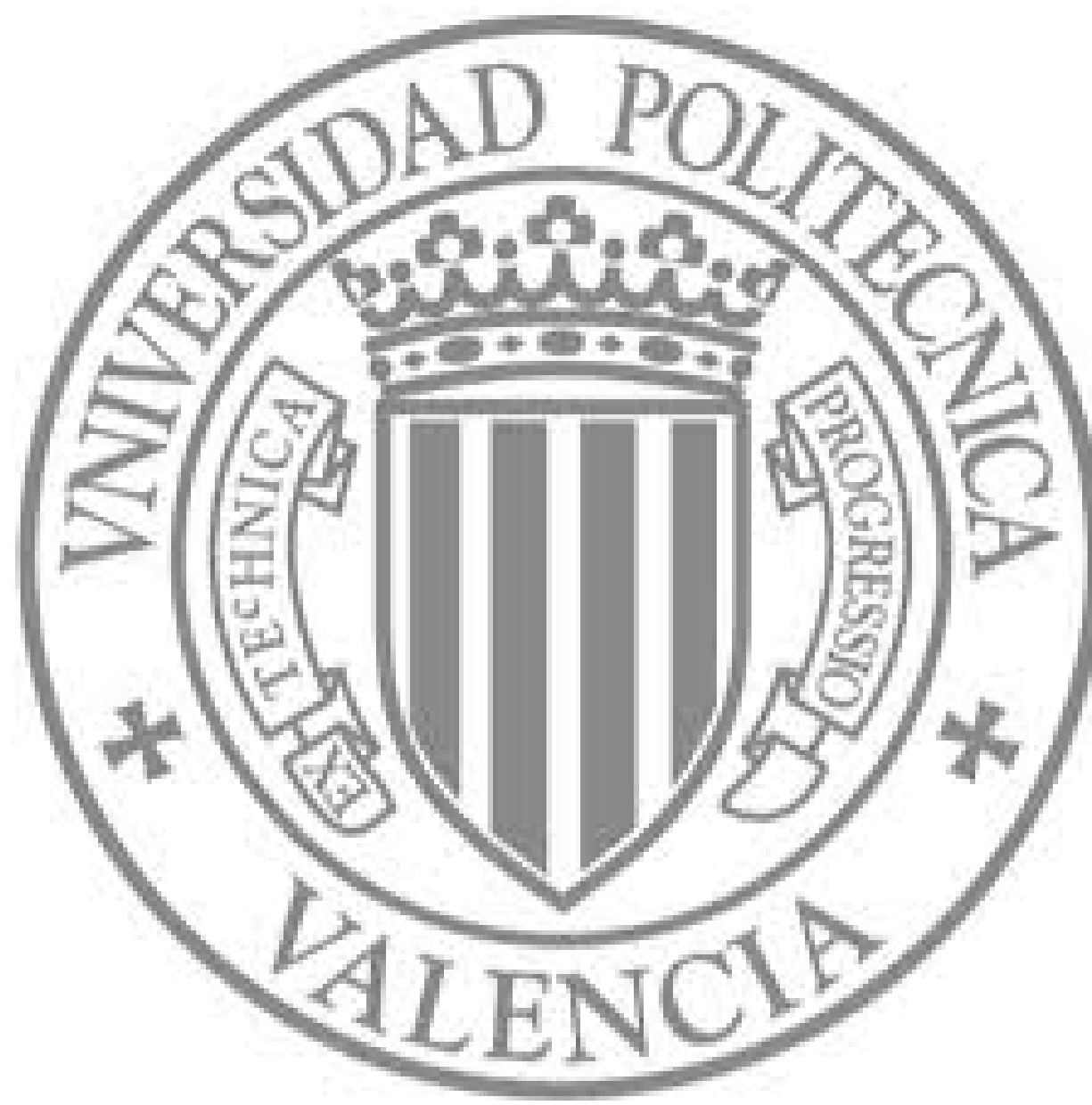

The final publication is available at

http://dx.doi.org/10.1179/1752270611Y.0000000010

Copyright Maney Publishing 


\title{
FRAME TRANSFORMATION AND GEOID UNDULATION TRANSFER TO GNSS REAL TIME POSITIONS THROUGH THE NEW RTCM 3.1 TRANSFORMATION MESSAGES
}

\author{
R. Capilla ${ }^{1}$, A. Martín', A. B. Anquela ${ }^{2}$ J. L. Berné \\ ${ }^{1}$ Cartographic Institute of Valencia, Spain \\ ${ }^{2}$ Department of Cartographic Engineering, Geodesy and Photogrammetry, Polytechnic \\ University of Valencia, Spain
}

\begin{abstract}
Radio Technical Commission for Marine Services (RTCM) standardized messages play an important role in real time Global Navigation Satellite Systems (GNSS) applications such as navigation, positioning, civil engineering, surveying, and cartographic or cadastral production. One of the latest agreements on RTCM definitions contains the data fields for real time geodetic reference frame transformation and orthometric heights computation by received geoid undulations via internet protocol. These parameters can be generated dynamically by a GNSS data center in a network of reference stations, encapsulated in RTCM messages and broadcasted to the rover location so they are centrally administered and the same frame transformations and geoid model are available to every user in the field, obtaining results in a local reference frame in real time. This paper summarizes the functionality of the new RTCM 3.1 transformation messages, describes limitations and provides ideas about the possible use for solving specific problems. Test field campaigns are used to describe the real performance and usefulness of these new RTCM 3.1 messages.
\end{abstract}

KEYWORDS. GNSS. Space frame-datum transformation. Geoid undulation transfer. RTCM messages. Real time.

\section{INTRODUCTION}

RTCM was originally set up as a common format for maritime standards, but nowadays it is applied world-wide to land and maritime positioning systems (www.rtcm.org). The RTCM subcommittee SC104 has released a revised RTCM 3.1 document [11], [12], which includes messages that are able to transfer geodetic transformations and geoidal information to the GNSS rover. The main advantage of this method is the central administration of these data which broadcasts consistent sets of streams with transformation parameters and geoid undulations to every user, therefore, pre-calculating transformation parameters and geoid model, usually set up in advance to GNSS controllers, becomes an unnecessary and obsolete task.

Definition and implementation of transformation messages in real time streams with several tools has been investigated and developed by Jäger and Kalber [7]. Based on these ideas, this paper describes the initial tests of this new concept for GNSS Network service providers. Frame transformation parameters and special grid residuals between European Terrestrial Reference Frame 1989 (ETRF89) and European Datum 1950 (ED50) have been implemented at the GNSS control center, and have been broadcasted to the rover location as an additional data field of RTCM 3.1 range of messages. At this point, an interesting possibility is the integration of several methods for geodetic transformations for real time NTRIP (Networked Transport of RTCM via Internet Protocol) transport: Classical geodetic transformations are sent together with dynamic generation of geodetic grids for networked transport in the same stream. Following the same idea, alignment with different International Terrestrial Reference Frame (ITRFxx) to ITRFyy or ETRFyy, global and regional frames, is treated as a 
geodetic transformation in order to give a solution for GNSS service providers who need to update station coordinates to different ITRFxx frames. Finally, dynamic grids for orthometric heights are computed, encapsulated and broadcasted using a local gravimetric geoid and the Earth Gravitational Model 2008 (EGM2008).

Field campaigns have been done for test purposes and some points, mainly related to tolerances of interpolated values between high residuals values in the networked transport of transformations, are discussed.

\section{REAL TIME AND DYNAMIC GENERATION OF RTCM TRANSFORMATION MESSAGES}

\section{Definition of the new transformation messages}

Definitions of the new range, 1021 to 1027 RTCM 3.1 Transformation Messages with the specifications for Coordinate Reference Systems (CRS), have the following characteristics according to RTCM Paper 100-2007-SC104, Amendment 1 to RTCM Standard 10403.1 [12]:

Message 1021 provides reserved data fields for three dimensional Bursa-Wolf geodetic transformation using strict formulae of the seven-parameter (7P) Helmert transformation or the 5-parameters of the abridged formulae of Molodensky transformation between ellipsoids.

Message 1022 provides the parameters for a Badekas-Molodensky geodetic transformation.

Message 1023 provides the dynamic generated residuals of a geodetic datum transformation by means of a grid model defined by ellipsoidal geodetic coordinates (in arc-seconds) and/or geoid undulations. .

Message 1024 provides the same residuals as message 1023 but using grids related to East-North CRS. 1023 and 1024 messages must be broadcasted in different streams if both grid definitions exist for transformation.

Messages 1025, 1026 and 1027 provide the CRS declaration for cartographic projections and their own characteristic parameters, such as the Lambert conformal conic projection, the oblique Mercator projection and up to seven more projections commonly required in national cartographic agencies around the world [1].

\section{Real time data flow and architecture}

Application and analysis of new standards are an interesting task for GNSS data center managers and researchers to provide efficient feedback and feed-forward for current and future definitions of real time standardizations for navigation and positioning. The desired reference transformations are set up within transformation modules or external tools and as a part of the Ntrip-Server of the Real Time Kinematics (RTK) corrections provider.

Bidirectional communication is required to use these messages effectively in Network RTK Solution, except for messages 1021 and 1022 where a broadcast link in one-way communication is possible. In the first case, the rover transmits its autonomous position solution coordinates (NMEA-GGA position, which is part of the NMEA 0183 set of standards of the National Marine Electronics Association) to the server, it is passed through the administrating GNSS networking software to the RTCM transformation messages module, and the server then broadcasts the frame/datum transformation and/or geoid undulation at the rover's location back to the field user, Figure 1. 


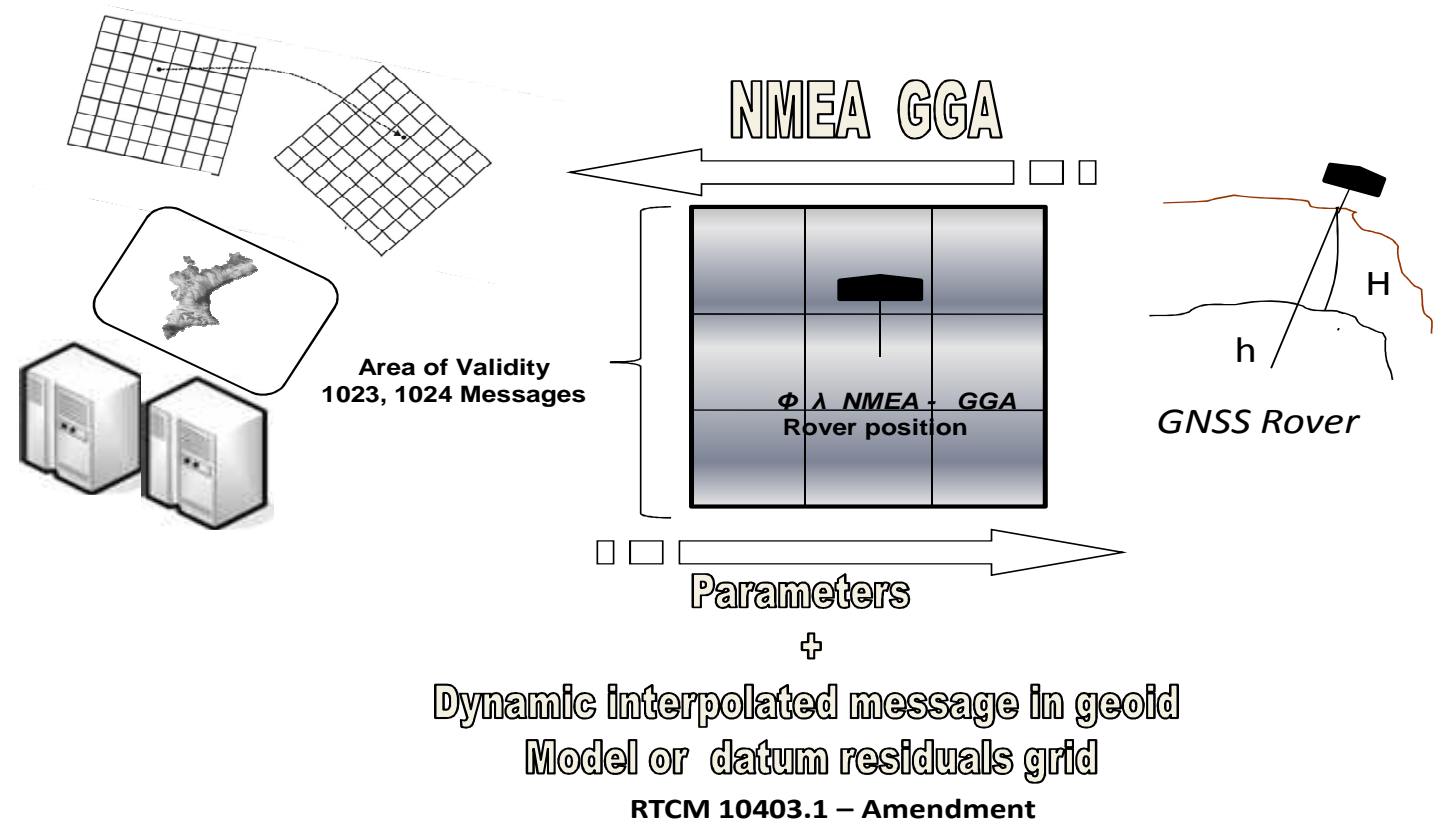

Fig. 1. Diagram of the data flow and architecture, for explanations see the text.

A critical step is the implementation, in the GNSS control center, of these geodetic transformations for use in real time transport. First of all, the definition of the area of validity must be specified at the network RTK processor. For 1021/1022 messages, the definition consists of the latitude and longitude coordinates of the origin and the extent of the area where the service must give support. Finally, messages belonging to the 1023-1024 range are computed using static grids with the extension of the area of the RTK service provider. In the server, a dynamic sub-grid is generated with the 4 or 16 nodes of these static grids surrounding the received rover NMEA position, which are used later for interpolation and define the area of validity (area of validity in Figure 1). The interpolation method is specified in one of the data fields of 1023 and 1024 messages (field controllers should be able to use any method supported in RTCM 3.1 definition, such as Bi-Linear, Bi-Quadratic, or Bi-Spline). The interpolated residual messages consist of 1, 2 or 3-dimensional shifts for geoid undulation or/and datum transformation.

Mean values in geodetic grid models and tolerances in residual interpolated values are established in the transformation messages RTCM 1023-1024 definition, which must be considered when using large extension and poor resolution grids for a whole country, Table 1 and Table 2. Shifts or jumps exceeding these tolerances, such as the result of interpolation for a rover position within cells, should be avoided. This question could be very critical in areas with a great geoid undulation gradient or in grids with a large separation between nodes (both geoidal or datum transformation). So, efficient dynamic generation of RTCM 3.1 messages depends, firstly, on the resolution and accuracy of known data in the source and target system for computing grid shift files, and secondly, on the capacity of real time dynamic residuals grid generation at the control centre for very large grids. 
Table 1. Current data Field Ranges/Tolerances in RTCM Paper 100-2007-SC104-STD for interpolated values in latitude, longitude, and geoid undulation grids

\begin{tabular}{c|cc}
\hline $\begin{array}{c}\text { Element of message } \\
\text { RTCM 3.1 }\end{array}$ & Data Field tolerance & Data Field Description \\
\hline Data Field 199 & $\begin{array}{c}\delta \varphi \mathrm{i} \pm 0.00765 \\
{[\text { in arc seconds }]}\end{array}$ & Residual in latitude for point i \\
Data Field 200 & $\begin{array}{c}\delta \lambda \mathrm{i} \pm 0.00765 \\
{[\text { in arc seconds }]}\end{array}$ & Residual in longitude for point i \\
& $\delta$ hi \pm 0.255 meters & Residual in interpolated $\mathrm{N}$ for point $\mathrm{i}$ \\
\hline
\end{tabular}

Table 2. Maximum and Minimum variations in nodes allowed in RTCM 3.1 transformation grids RTCM Paper 100-2007-SC104-STD

\begin{tabular}{c|c}
\hline $\begin{array}{c}\text { Element of message } \\
\text { RTCM 3.1 }\end{array}$ & $\begin{array}{c}\text { Max./Min. Displacement } \\
\text { allowed }\end{array}$ \\
\hline Data Field 205-206 & $\Delta \lambda, \Delta \varphi \pm 0.127^{\prime}$ \\
Data Field 207 & $\Delta$ Ngeoid \pm 163.8 meters \\
\hline
\end{tabular}

Char, bit or integer data fields are broadcast in transformation messages to specify interpolation method, target and source CRS parameters or the available European Petroleum Survey Group (EPSG) code. A unique System Identification Number (SIN) has to be used for all messages related to the same sets of parameters. As Amendment 1 of RTCM 10403.1 [12] defines, a SIN is necessary if more than one transformation method between two CRS's is transferred within the data streams, [7]. Every transformation can also be broadcast using one TCP-port.

Implementation of transformation Messages 1021-1024 for frame/datum
transformation transfer

Two different approaches have been tested for ETRF89-ED50 transformation. First of all a linear expression of 7P Bursa-Wolf model, computed using more than 1500 points with precise coordinates in ETRF89 and ED50, has been implemented in the transformation message 1021 , with an area of validity of $25000 \mathrm{~km}^{2}$ on the East Coast of Spain. These points belong to the Geodetic Network of the Region of Valencia (Spain), and the density of the geodetic network is approximately 1 point for every 2.5 $\mathrm{km}$, Figure 2 [5]. The second approach is the real time transmission of the National Datum transformation between ETRF89 and ED50 for Spain computed by the National Geographic Institute of Spain (IGN). This transformation adopts the binary format of NTv2 (National Transformation Version 2 grid file) as described in [6]. As European Datum 1950 accuracy and residual propagation or distortion (difference between 7P transformed coordinates from ETRF89 to ED50 and "real" ED50 coordinates) of the network throughout the country is heterogeneous, the classic and unique set of seven parameters is not accurate enough to guarantee a consistent transition between ETRF89 and ED50 throughout the Iberian Peninsula. As an efficient and successful alternative, grid node values in the NTv2 grid (IGN) are obtained by modelling the distortion with the minimum curvature surfaces technique. 


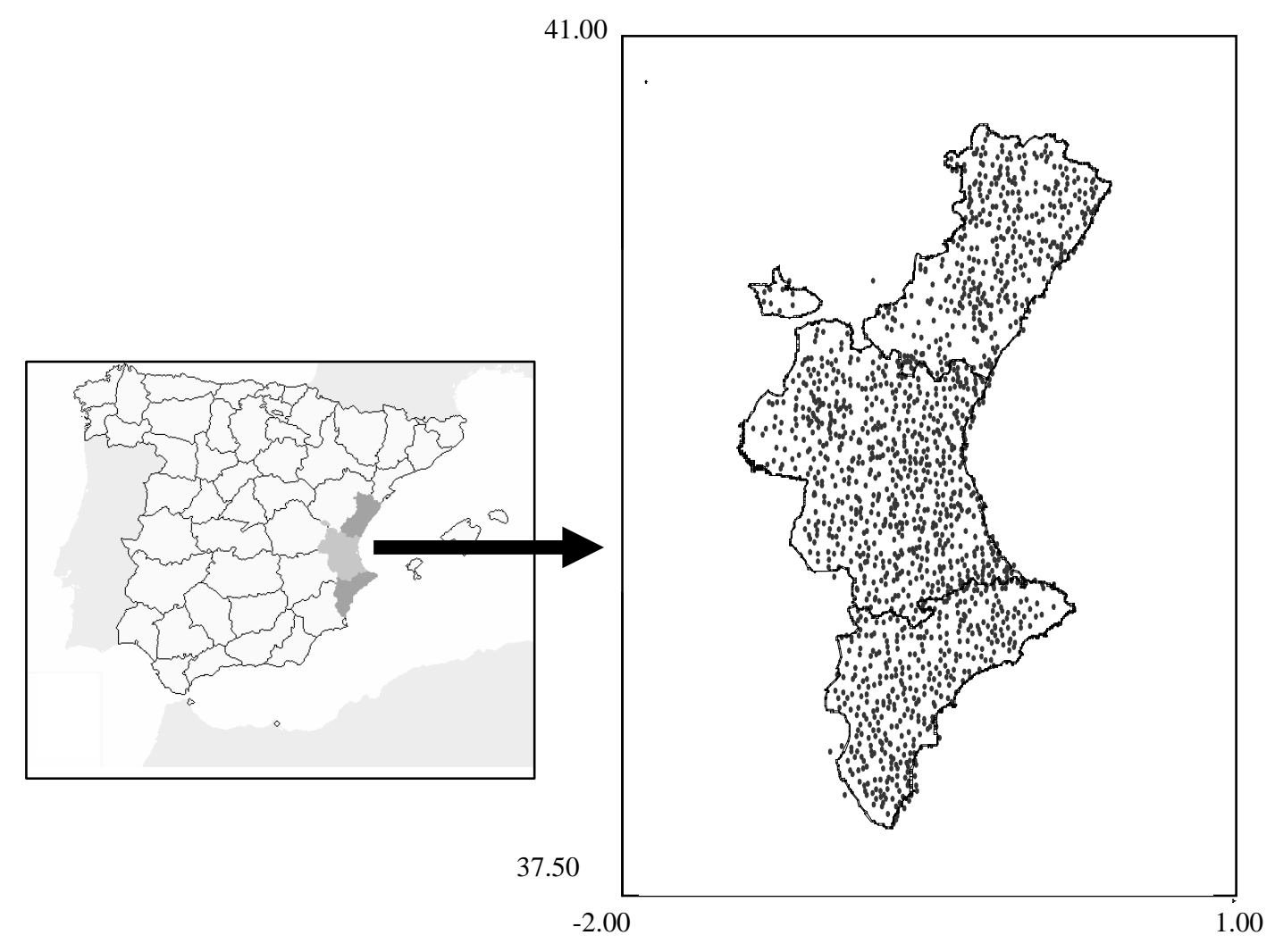

Fig. 2. Passive geodetic infrastructure of Valencia.

In the NTv2 grid, the mean displacement grid shifts, $\Delta \lambda, \Delta \varphi$, between Geodetic Reference Systems ETRF89 and ED50, reach values of 4-5 in arc-seconds, and residual interpolated values in the NTv2 grid for messages 1023 or 1024 exceed the established tolerance values of Table 1 and 2. Consequently, they cannot be used for real time transport. A new grid that follows the specifications of RTCM 3.1 standards is necessary. Therefore, an attractive possibility for the dynamic generation of transformation parameters appears which consists in encapsulating the classic seven parameter transformation into one RTCM 3.1 message (1021), and use 1023 or 1024 message for sending the residual distortion by means of a new grid. Both messages can be broadcast in the same stream.

In the case of the NTv2 grid, the differences between the application of the classic conformal transformation of 7P, and the application of the NTv2, are re-assigned to the nodes of a new grid and are encapsulated in message 1023. The new grid shifts do not exceed the tolerances of Tables 1 and 2 and they only transport the residual distortion. Final residuals reach values of minimum -0.03 " or maximum 0.005 ".

Obviously, this procedure can be avoided if the new grid can be computed with the same original process used to compute NTv2 values, so it would contain the modelling distortion component of the transformation with the minimum curvature surface technique.

Finally, a third point involving the development of RTCM 3.1 transformation messages is the following: positioning service providers must distribute and use products consistent with International GNSS Service (IGS) standards, and the network must be constrained to ITRFxx frame. The treatment of transformation from ITRF global frames to regional frames has been provided in geodetic literature [13]. Furthermore, the frames defined by International Earth Rotation and Reference Systems, that is, station coordinates and velocities, are updated about every five years. If a network administrator updates its coordinates to the last frame, or if the GNSS 
network adopts dynamic reference frames, it could produce jumps in station positions or discontinuities that are not practical for end users, [2]. So, a NTRIP stream with a 1021 message could be implemented in order to broadcast to users the seven-parameter transformation that aligns updated GNSS reference station coordinates with the previous solution in ETRF89 coordinates (obtained from previously adopted ITRFxx). To check this point, estimated offsets between ITRF2005 and Regional Reference Frame Sub-Commission for Europe (EUREF) recommended ETRF2000 common frame, [3], have been encapsulated in RTCM 3.1 1021 message.

\section{Implementation of transformation messages 1023-1024 for geoid undulation transfer}

Two different geoid models are used for real time dynamic generation of undulations: the recently developed gravimetric high-precision and high-resolution geoid model for the region of Valencia, GECV07, [8], based on the remove-restore technique using the Stokes-Helmert approach, and the Earth Gravitational Model 2008, EGM2008 [10], a spherical harmonic development complete to degree and order 2159 with additional coefficients extending to degree 2190 and order 2159. An analysis of this model compared with local and regional solutions can be found in [9].

The geoidal information for both geoid models can be generated and encapsulated in RTCM 1023-1024 messages. The original sampling interval of the GECV07 grid is 2 minutes, both in latitude and longitude, and the residual interpolated values for message 1023 or 1024 do not exceed the established tolerance for height values according to Table 1 . However, in order to carry out the task with that tolerance, the undulation values for the EGM2008 solution have to be computed in a grid without a large separation between nodes, 1 minute both in latitude and longitude has been adopted.

\section{FIELD TESTS}

Two different datasets are used to describe the performance of RTCM3.1 messages generated in different streams following the specifications explained in the previous section: a set of 15 points that belong to the region's Geodetic Network, Figure 3, and the seven points of the Polytechnic University of Valencia's calibration geodetic line, Figure 4. This calibration geodetic line is very useful when testing the "navigation" or movement of a rover between the calibrated points, inside the same area of validity of networked grid transformations. In the test areas, the NTRIP services for real time positioning are provided by the GNSS Reference Station Network of Valencia (known as the ERVA Network). This network has been executed and administrated by the Cartographic Institute of Valencia (ICV), [4], [5]. 

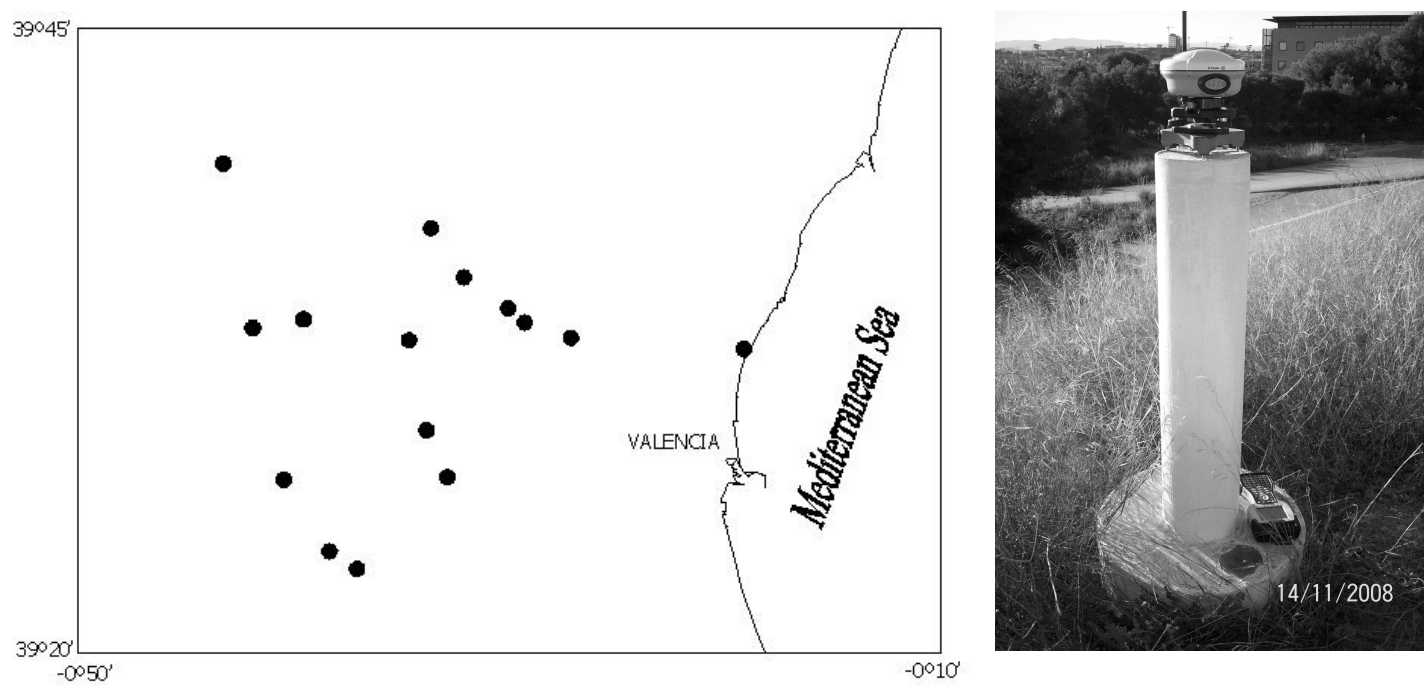

Fig. 3. Location of the geodetic points for the field test.
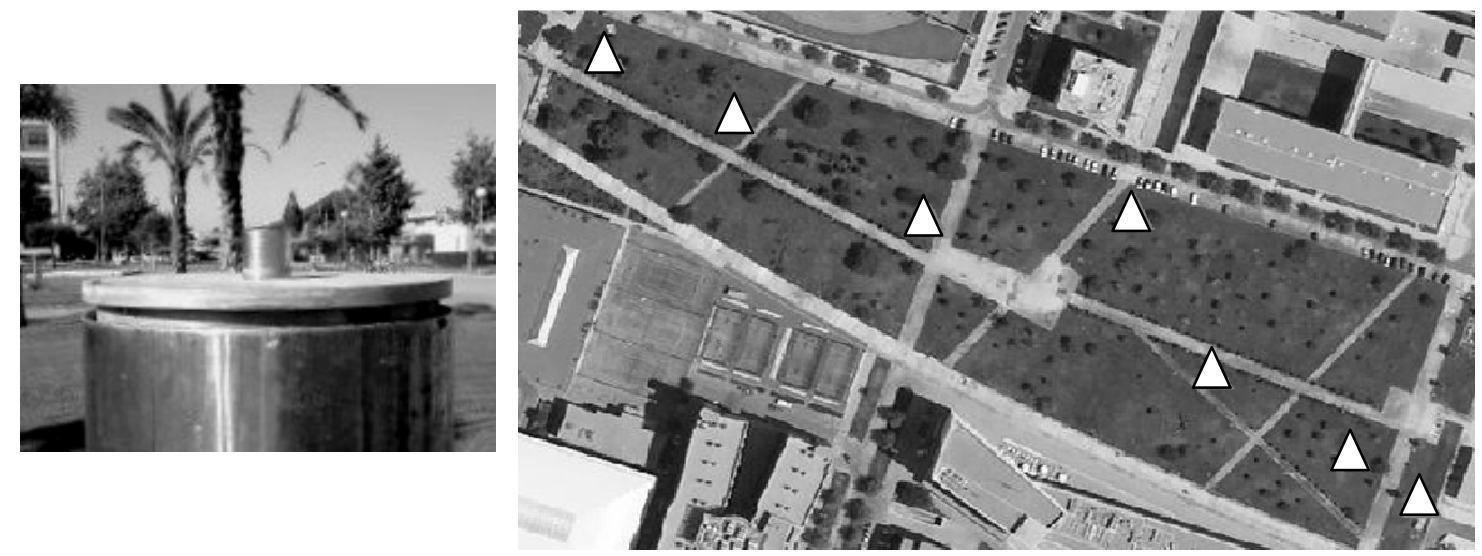

Fig. 4. Calibration geodetic network of the Polytechnic University of Valencia.

An important practical aspect to bear in mind is that the correct solution for the application of range messages 1021, 1023 depends on the real time accuracy of the planimetric and altimetric obtained position. That is to say that poor quality GNSS observation or latency distorts the transformed position at the rover's location, so real time measurements were done with a real time latency $<1$ second. All field test results are derived from RTK network corrections received every second Observation periods were planned in advance with optimal constellation conditions, all real time results with less than 7 NAVSTAR-GPS constellation were rejected. Both datasets obtained in the geodetic network and calibration geodetic line, were measured by means of five stored occupations in every point with 120 epochs-seconds observation time span in every real time occupation. Mean time-to-fix for real time ambiguities was about 20 seconds.

Following the guidelines for bidirectional links and transmission of transformation messages, a network RTK processor was set up to broadcast residuals messages every 5 epochs [12].

Table 3 shows the statistics for the field test results in the 15 geodetic points involving the application of ETRF89-ED50 transformation with real time broadcast 7P 1021 message. The first row, called network RTK accuracy, is the real time accuracy 
in the measured position. The second, called transformation transport error, is the difference between the transformation of the ETRF89 RTK measured position with stored parameters in the controller and the RTK transformed position received (applying RTCM 3.1 transformation messages to the RTK measurements using the same parameters that are stored in the controller). The third row, called final error, is the difference between transformation of the ETRF89 coordinates (adjusted solution of the Geodetic Network) and RTK transformed position received by applying RTCM 3.1 transformation messages, that is, accuracy of broadcasted transformation + accuracy in positioning.

As can be seen, the mean transformation transport error is less than RTK accuracy in the measured position (as expected), so the final error becomes the difference between RTK observed position solution and ETRF89 coordinates, which means that no transformation or latency error in the transport can be assumed.

Table 3. Statistics of the differences in the 1021 transformation message test, see text for explanations. Units in meters

\begin{tabular}{l|cccc}
\hline \multicolumn{1}{c|}{$\begin{array}{c}\text { Mean PDOP:1.6 } \\
\text { Mean Latency:0.9 sec }\end{array}$} & Mean & $\sigma$ & Max. & Min. \\
\hline Network RTK accuracy & 0.009 & 0.002 & 0.014 & 0.006 \\
Transformation transport error & 0.003 & 0.003 & 0.017 & 0.000 \\
Final error & 0.024 & 0.013 & 0.063 & 0.007 \\
& & & & \\
\hline
\end{tabular}

Table 4 shows the statistics for the field test results, in the 7 points of the calibration line, of the ETRF89-ED50 transformation 1021 and 1023 messages following the separation approach for standardization in RTCM of national datum transformation NTv2 grid with distortion modelling. The first row, called complete transformation transport error, is the difference between the transformation using the original NTv2 grid applied to the RTK obtained position and RTK transformed position received by applying RTCM 3.1 messages 1021 (conformal transformation) + 1023 (residual distortion modelling). The second row, called complete final error, is the difference between transformation using the original NTv2 grid applied to the ETRF89 coordinates (adjusted solution of the geodetic network) and RTK transformed position received by applying RTCM 3.1 transformation messages 1021+ 1023 grid.

As can be seen, the greater part of the complete final error becomes the difference between network RTK solution and ETRF89 coordinates, so no transformation or real time interpolation errors exist in the treatment of the original NTv2 grid.

Table 4. Statistics of the differences in the 1021 and 1023 transformation messages test, see text for explanations. Units in meters

\begin{tabular}{l|cccc}
\hline \multicolumn{1}{c|}{$\begin{array}{c}\text { Mean PDOP:1.6 } \\
\text { Mean Latency:0.7 sec }\end{array}$} & Mean & $\sigma$ & Max. & Min. \\
\hline Complete transformation transport error & 0.000 & 0.003 & 0.003 & -0.002 \\
Complete final transport error & 0.002 & 0.007 & 0.010 & -0.002 \\
\hline
\end{tabular}


The same conclusions are obtained for the ITRF05-ETRS89/ETRF05 and ITRF00ETRS89/ETRF00 frame alignment, using transformation message 1021 for broadcasting estimated offsets between frames. If offsets present mean values that are larger than RTK positioning with network solution accuracy, these offsets imply a jump in the RTK solution. This is the case of coordinate jumps for reference stations computed in different frames and also using different antenna calibration models (from previous relative models to actual absolute antenna phase centre calibrations). Table 5 presents the statistics for the field test results in the 15 geodetic points of the application of the mean translation between ERVA08 and ERVA05 network solution.

Table 5. Statistics of the differences in application of mean translation between ERVA08-ERVA05 network solution. Units in meters

\begin{tabular}{l|cccc}
\hline \multicolumn{1}{c|}{$\begin{array}{l}\text { Mean PDOP: } 1.3 \\
\text { Mean Latency:0.8 sec }\end{array}$} & Mean & $\sigma$ & Max. & Min. \\
\hline Transformation transport error & -0.003 & 0.008 & 0.006 & -0.014 \\
Final error & 0.003 & 0.009 & 0.015 & 0.012 \\
& & & & \\
\hline
\end{tabular}

Table 6 shows the statistics for the field test results for the 15 geodetic points introducing the GECV07 geoid model in transformation message 1023. The first row, called planimetric network RTK accuracy, is the real time planimetric accuracy in the observed position with network solution. The second row, called altimetric network RTK accuracy position, is the real time altimetric accuracy in the observed position with the same technique. The third row, transformation transport error, is the difference between the postprocessing application of computed geoid undulations at the observed RTK ellipsoidal height and real time geoid undulations received by applying RTCM 3.1 transformation messages. The fourth row, final error, is the difference between the application of computed geoid undulations in the ETRF89 ellipsoidal height (adjusted solution) and real time geoid undulations received by applying RTCM 3.1 transformation messages.

As can be seen, the planimetric RTK accuracy position is less than 0.02 meters, so the error in the geoid undulation due to an error in planimetric position can be ignored. The third row presents similar results to the altimetric RTK accuracy position in terms of the sum of mean and $\sigma$ values or the sum of Maximum and Minimum values (as expected because an uncertainty in altimetric position, due to the dispersion of ellipsoidal height determination in GPS measurements, becomes an error in the transformed orthometric height). Obviously the greater part of the final error becomes, in this case, the transformation transport error. 
Table 6. Statistics of the differences in the GECV07 geoid test. See text for explanations. Units in meters

\begin{tabular}{l|cccc}
\hline \multicolumn{1}{c|}{$\begin{array}{c}\text { Mean PDOP:1.6 } \\
\text { Mean Latency:0.7 sec }\end{array}$} & Mean & $\sigma$ & Max. & Min. \\
\hline Planimetric network RTK accuracy position & 0.009 & 0.002 & 0.013 & 0.006 \\
Altimetric network RTK accuracy position & 0.014 & 0.004 & 0.025 & 0.008 \\
Transformation transport error & -0.002 & 0.010 & 0.015 & -0.027 \\
Final error & -0.001 & 0.011 & 0.018 & -0.024 \\
\hline
\end{tabular}

Finally, Table 7 shows the statistics for the field test results in the 7 points of the calibration geodetic line introducing the EGM2008 geoid undulation in transformation message 1023. The first and second rows show the transformation transport accuracy and final error, respectively, with the same meaning and conclusions as in the GECV07 test.

Table 7. Statistics of the differences in the EGM2008 geoid test, See text for explanations. Units in meters

\begin{tabular}{l|cccc}
\hline \multicolumn{1}{c|}{$\begin{array}{c}\text { Mean PDOP: } 1.3 \\
\text { Mean Latency:0.8 sec }\end{array}$} & Mean & $\sigma$ & Max. & Min. \\
& & & & \\
\hline Transformation transport error & -0.003 & 0.009 & 0.000 & -0.011 \\
Final error & 0.001 & 0.009 & 0.006 & -0.002 \\
\hline
\end{tabular}

\section{CONCLUSIONS}

This paper has introduced a brief description of the use of RTCM 3.1 transformation messages in a GNSS Central Network Processor. Use in precise real-time applications of new and updated high-resolution geoid models and national grids for datum transition or frame translations is possible in the RTCM streams by means of external or embedded transformation modules, following the standardization of RTCM 3.1 messages as explained in the article.

In the studied context, these new messages are used for real time transport of ETRF89 to ED50 datum transformation (using message 1021 or 1021+1023), for frame alignments in a GNSS Network and for ellipsoidal to orthometric height conversion (using local GECV07 geoid model or global EGM2008 geopotential model). The field tests show good performance and agreement in the application of networked transport of transformation messages, so real time kinematic users of the GNSS network can develop their work with these new real time products avoiding the step of introducing datum transformation and geoid models in the field controllers.

Implementation and preparation of geodetic grid models for efficient dynamic generation of these messages must be done and should be an additional task for GNSS Network providers and data centers, but the work will ensure that end users of real 
time positioning services have access to broadcast transformations in the NTRIP streams and can work with the updated and centralized information.

The evaluation of future RTCM standardizations and definitions is expected to continue in order to consider Earth-Centered, Earth Fixed (ECEF) Plate Fixed transformation for future messages (1028) and relations between global frames of IGS real time broadcasted products (orbits) or between global and regional frames (ETRF89). GNSS positioning methods with great possibilities like Precise Point Positioning (PPP), are based on the aim of providing precise knowledge and standardized transmission of individual error sources by means of State Space Representation technique, [14], [15], and will need to be completed with real time information diffusion of reference frames.

\section{ACKNOWLEDGEMENTS}

The authors wish to thank to Reiner Jäger for providing information on the new RTCM messages and implementation, Leica Geosystems in Spain and Trimble Iberica for their help with the new firmware versions and their interest in the research. We also appreciate the efforts of Mayte Zaragozá and Roser Soler for their assistance in the field campaigns. Finally, the authors wish to thank J. González-Matesanz from IGN.

\section{References}

1. Annoni, A., Luzet, C., Gubler, E., Ihde J. 2003. Map Projections for Europe, EC-JRC-Eurogeographics, European Communities.

2. Benciolini, B., Biagi, M., Crespi, M., Manzino, A.M., Roggero, M. 2008. Reference frames for GNSS positioning services: Some problems and proposed solutions, Journal of Applied Geodesy, 2, 53-62

3. Boucher, C., Altamimi, Z. 2008. Memo: Specifications for reference frame fixing in the analysis of a EUREF GPS campaign, EUREF IAG Subcomission.

4. Capilla, R., Blat-Puchades, E., Saa-Gonzalez, J.M., Bretos, J.J. 2005. Arquitectura y servicios de la red de Estaciones GPS/GNSS de Valencia, $V$ Asamblea Hispano-Portuguesa de Geodesia y Geofisica, Sevilla.

5. Capilla, R., Die, J., Esteso, E. 2006. Diseño, aplicaciones integradas y rendimiento de la red activa GPS/GNSS de Valencia, VII International Geomatic Week, Barcelona.

6. González-Matesanz, J., Dalda, A., Malpica, J.A. 2006. A range of ED50ETRS89 datum transformation models tested on the Spanish geodetic network, Survey Review, 38(302), 654-667.

7. Jäger, R., Kälber, S. 2008. The new RTCM 3.1 transformation messages declaration, generation from reference transformations and implementation as a server-client concept for GNSS services. International Conference GEOS 2008.

8. Martín, A., Capilla, R., Anquela, A.B., Padín, J., Berné, J.L. 2008. Highprecision and high-resolution gravimetric geoid determination in Valencia (Eastern Spain), I International congress on geomatic and surveying engineering, Proceedings, Valencia.

9. Martín, A., Anquela, A.B., Padín, J., Berné, J.L. 2009. Hacia una nueva era en la determinación de modelos de geoide. Análisis del nuevo modelo global EGM2008, VIII International Geomatic Week, Barcelona.

10. Pavlis, N.K., Holmes, S.A., Kenyon, S.C., Factor, J.K. 2008. An Earth gravitational model to degree 2160: EGM2008, European Geosciences Union General Assembly, Vienna 2008. 
11. RTCM Special Committee No. 104. 2006. RTCM Standard 10403.1 for differential GNSS (Global Navigation Satellite Systems) Services - Version 3, Radio Technical Commission for Maritime Services.

12. RTCM Special Committee No. 104. 2007. Amendment 1 to RTCM Standard 10403.1 for differential GNSS (Global Navigation Satellite Systems) Services Version 3, Radio Technical Commission for Maritime Services.

13. Soler, T, Marshall, J., 2003. A note on frame transformations with applications to geodetic datums, GPS Solutions, 7(2), 148-149.

14. Wübbena, G. 2007. RTCM State Space Working Group-Mission Statement, RTCM Paper 075-2007-SC104-470

15. Wübbena, G. 2008. GNSS Network-RTK Today and in the Future Concepts and RTCM Standards. International Symposium on GNSS, Space-based augmentation systems and applications, Berlin. 